\title{
Downlink Electric-Field and Uplink SAR Prediction Algorithm in Indoor Wireless Network Planner
}

\author{
D. Plets*, W. Joseph*, K. Vanhecke*, L. Martens* \\ * Information Technology Department, Ghent University/iMinds \\ Gaston Crommenlaan 8, B-9050 Ghent, Belgium \\ david.plets@intec.ugent.be
}

\begin{abstract}
A heuristic network calculator for both downlinkand uplink-induced exposure in indoor wireless networks is applied to two indoor environments for three phone call scenarios: UMTS macrocell, UMTS femtocell, WiFi voice-overIP. The electric-field strength due to downlink and localized SAR distributions due to uplink are evaluated. Dependent on the building location relative to existing macrocells and on the user's phone call duration, different configurations might be preferential from an exposure point of view.
\end{abstract}

Index Terms-SAR, exposure, WiFi, UMTS, femtocell, electricfield strength

\section{INTRODUCTION}

Current wireless network calculators or planners (e.g., [1], [2]) rarely account for downlink (DL) exposure in wireless networks (electric-field E originating from the base stations or access points (APs)), and to the author's knowledge, never for uplink-induced (UL) localized exposure (localized Specific Absorption Rate (SAR) due to the mobile device's transmitted signal). In this paper, an existing network planner (WHIPP [3]) will be extended with prediction algorithms for the simulation and visualization of the electric-field strengths due to DL traffic and localized SAR values due to UL traffic. Three phone call scenarios will be defined and compared with respect to DL exposure and UL exposure: a user device connecting to $\mathrm{a}$ (n outdoor) Universal Mobile Telecommunications System (UMTS) macrocell, to an indoor UMTS femtocell network, and to an indoor WiFi AP network. The algorithms will be applied to two indoor environments. The first is the third floor of an office building, the second one is an open test lab.

\section{Methodology}

\section{A. Coverage model}

For the coverage prediction and network planning, the WiCa Heuristic Indoor Propagation Prediction (WHIPP) tool is used, a set of heuristic planning algorithms, experimentally validated for network planning in indoor environments [3]. The path loss prediction algorithm takes into account the effect of the environment on the wireless propagation channel and bases its calculations on the determination of the dominant path between transmitter and receiver, i.e., the path along which the signal encounters the lowest obstruction. The WHIPP tool is designed for optimal network planning with a minimal number of access points (AP) [3]. It also allows predicting the electricfield and the localized $\mathrm{SAR}_{10 \mathrm{~g}}^{\max }$ values, as will be explained in the remainder of this paper.

\section{B. Wireless equipment}

For the WiFi AP and UMTS femtocell scenario, the WHIPP planning tool will first design a network according to the WiFi (1 Mbps DL and UL) and UMTS (12 kbps DL and $\mathrm{UL})$ coverage requirements in the different rooms, the $\mathrm{WiFi}$ and UMTS voice call receiver sensitivities and transmit powers of both the AP and the considered mobile phone MP (type Nokia N95), and the network planner's path loss models [3]. For the UMTS macrocell scenario, the mobile phone connects to an outdoor UMTS macrocell. Since the WHIPP tool is specifically developed for indoor environments, it will not be used for the macrocell exposure calculations. Therefore, electric-field and SAR values will be determined from measurements inside the building. Based upon these measurements and on the UMTS power control principle, a simulation of other locations of the macrocell base station (relative to the considered office building) will be investigated, four in total.

a) Downlink: In this paper, base stations with an Equivalent Isotropically Radiated Power (EIRP) of $10 \mathrm{dBm}$ are assumed, operating at a frequency of $2151.6 \mathrm{MHz}$ for DL UMTS, and $2412 \mathrm{MHz}$ for DL WiFi. At the receiver side, a mobile phone with an assumed UMTS receiver sensitivity of $-95.1 \mathrm{dBm}$ is used, as derived in [4]. The assumed WiFi receiver sensitivity is $-98.4 \mathrm{dBm}$, based on the specifications of a typical $802.11 \mathrm{~b} / \mathrm{g}$ receiver chipset. The assumed values are summarized in Table I.

\begin{tabular}{|c|c|c|c|c|}
\hline & $\begin{array}{r}\text { required } \\
\text { received } \\
\text { throughput }\end{array}$ & $\begin{array}{r}\text { receiver } \\
\text { sensitivity } \\
{[\mathrm{dBm}]}\end{array}$ & $\begin{array}{r}\text { transmit } \\
\text { EIRP } \\
\text { [dBm] }\end{array}$ & $\begin{array}{r}\mathbf{S A R}_{10 \mathrm{~g}}^{\max } \\
{[\mathrm{W} / \mathrm{kg}]}\end{array}$ \\
\hline phone UMTS & $12.2 \mathrm{kbps}$ & -95.1 & variable & 0.415 \\
\hline phone WiFi & $1 \mathrm{Mbps}$ & -98.4 & 20 & 0.049 \\
\hline femtocell UMTS & $12.2 \mathrm{kbps}$ & -110 & 10 & - \\
\hline access point $\mathrm{WiFi}$ & $1 \mathrm{Mbps}$ & -98.4 & 10 & - \\
\hline
\end{tabular}

TABLE I

WIRELESS EQUIPMENT CHARACTERISTICS.

b) Uplink: The mobile phone's maximum spatial peak SAR values in a $10 \mathrm{~g}$ cube $\left(\mathrm{SAR}_{10 \mathrm{~g}}^{\max }[\mathrm{W} / \mathrm{kg}]\right)$ are obtained 
from certified compliance measurements [5], [6] with the phone that was used for validation of the models [4] and will be used here for simulation. For the $1800 \mathrm{MHz}$ band (UMTS), a $\mathrm{SAR}_{10 \mathrm{~g}}^{\max }$ value of $0.415 \mathrm{~W} / \mathrm{kg}$ for an antenna EIRP of $0.2 \mathrm{~W}$ was obtained [4], [6]. The $\mathrm{SAR}_{10 \mathrm{~g}}^{\max }$ value will also be used to calculate localized SAR values for the macrocell scenario, since this value is essential to convert device transmit power values to SAR values. For the $2400 \mathrm{MHz}$ band, an $\mathrm{SAR}_{10 \mathrm{~g}}^{\max }$ value of $0.049 \mathrm{~W} / \mathrm{kg}$ was reported in [5] for an antenna EIRP of $0.1 \mathrm{~W}$ and a duty cycle of $100 \%$. The receive sensitivities of the UMTS femtocell base station and WiFi AP are set at $-110 \mathrm{dBm}$ (after calibration [4]) and $-98.4 \mathrm{dBm}$ (same WiFi chipset as for the WiFi receiver inside the mobile phone) respectively. The UL frequency from mobile phone to femtocell base station (FBS) is 1957.6 MHz. The considered values are summarized in Table I.

\section{Simulation Environments}

For the three considered connection scenarios, we will investigate the electric-field and SAR distributions inside two different buildings. The first one is the office building depicted in Fig. 1. The building is $90 \mathrm{~m}$ long and $17 \mathrm{~m}$ wide and consists of concrete walls (grey) and layered drywalls (brown). Fig. 2 shows the layout of the second building, a test lab at the university. It is an open room (66 m x $20.5 \mathrm{~m})$, consisting of 60 nodes in a grid configuration with an $\mathrm{x}$-separation of $6 \mathrm{~m}$ and a y-separation of $3.6 \mathrm{~m}$. The 60 installed nodes are represented by the blue locations in Fig. 2 .

\section{Simulation MODELS}

The exposure values for the different connection scenarios will be separately calculated for UL traffic and DL traffic. DL exposure will be expressed as a function of the electricfield strength generated by the incident waves from the base station (macrocell, femtocell, or WiFi AP). UL exposure will be expressed by a localized $\mathrm{SAR}_{10 \mathrm{~g}}$ value in the head due to transmission from user device to the base station. The UMTS indoor model used for the simulations was validated with an accuracy of $3 \mathrm{~dB}$ or better for UMTS in [4] and the WiFi model was successfully validated in [3].

\section{A. Downlink: Electric-field model}

The electric-field strength $[\mathrm{V} / \mathrm{m}]$ (at the location of the mobile phone MP) due to an indoor transmitting source (UMTS FBS or WiFi AP) is calculated based upon the transmitting source's EIRP and the path loss predicted by the WHIPP tool, as derived in [7]. WHIPP simulations have already been validated with measurements in [7]. For WiFi APs, the calculated electric-field strength is multiplied by the square root of the duty cycle (here assumed $2 \%$, based on measured 'Skype voice' duty cycles in [8]).

For the prediction of the electric-field strength due to the UMTS macrocell, we use actual received power measurements. These values will be presented in Section IV.

\section{B. Uplink: Localized SAR model}

For the calculation of the localized $\mathrm{SAR}_{10 \mathrm{~g}}[\mathrm{~W} / \mathrm{kg}]$, the following equation is used [9], [10].

$$
\mathrm{SAR}_{10 \mathrm{~g}}=\frac{\mathrm{P}_{\mathrm{Tx}}}{\mathrm{P}_{\mathrm{Tx}}^{\max }} \cdot \mathrm{SAR}_{10 \mathrm{~g}}^{\max },
$$

where $\mathrm{P}_{\mathrm{Tx}}[\mathrm{W}]$ is the power emitted by the user device, $\mathrm{P}_{\mathrm{Tx}}^{\max }[\mathrm{W}]$ is the maximal power emitted by the user device, and $\mathrm{SAR}_{10 \mathrm{~g}}^{\max }[\mathrm{W} / \mathrm{kg}]$ is the maximum spatial peak SAR in a $10 \mathrm{~g}$ cube, a value measured in a standard configuration [6].

For the considered device, a Nokia N95, SAR $\max _{10 \mathrm{~g}}$ for a radiated power $\mathrm{P}_{\mathrm{Tx}}^{\max }$ of $23 \mathrm{dBm}$ is $0.415 \mathrm{~W} / \mathrm{kg}$ (see Table I). The value $\mathrm{P}_{\mathrm{Tx}}^{\max }$ of $23 \mathrm{dBm}$ for UMTS is also stated in [9] and is confirmed by measurements with the device. For WiFi, $\mathrm{SAR}_{10 \mathrm{~g}}^{\max }$ for a UMTS radiated power $\mathrm{P}_{\mathrm{Tx}}^{\max }$ of $20 \mathrm{dBm}$ is 0.049 $\mathrm{W} / \mathrm{kg}$ (see Table I).

In order to predict the localized SAR values, an accurate prediction of the emitted power $\mathrm{P}_{\mathrm{Tx}}$ is required (see equation (1)). For UL WiFi traffic, $\mathrm{P}_{\mathrm{Tx}}$ will be assumed equal to the product of $\mathrm{P}_{\max }(20 \mathrm{dBm}$ or $0.1 \mathrm{~W})$ and the duty cycle $(2 \%$ [8]) (no power control).

For UL UMTS to femtocell, the mobile phone's emitted power $\mathrm{P}_{\mathrm{Tx}}$ will be predicted by the WHIPP tool (limited between $-57 \mathrm{dBm}$ and $23 \mathrm{dBm}$ [9]). $\mathrm{P}_{\mathrm{Tx}}$ will depend on the connection quality and the base station sensitivity. $\mathrm{P}_{\mathrm{Tx}}$ is modeled as the sensitivity $\mathrm{P}_{\text {sens }}$ of the UMTS FBS for maintaining a UMTS phone call (here set at $-110 \mathrm{dBm}$ [4]) minus the path loss PL between base station and user device (predicted by the WHIPP tool [3]). These models were validated in [4].

For UL UMTS to macrocell, we will again start from actual DL power values from and UL power values to the existing macrocell, measured in the considered building of Fig. 1. Based on these values, other macrocell base station locations are simulated by varying the received power in steps of $10 \mathrm{~dB}$. Due to the power control mechanism, these DL simulations will allow also determining the corresponding UL power. $\mathrm{SAR}_{10 \mathrm{~g}}$ values will then be calculated from this UL power.

\section{RESULTS AND DISCUSSION}

In the UMTS macrocell scenario, the mobile phone connects to an outdoor UMTS macrocell and E and SAR values are determined from measurements inside the building [4]. It was shown in [4] that it is fair to assume that the received and transmitted powers are uniformly distributed, with the same E and SAR value for each location inside the building.

Based on [9], it was also shown in [4] that different macrocell scenarios can be simulated by relating a $1 \mathrm{~dB}$ higher received signal power (higher $\mathrm{E}$ ) to a $1 \mathrm{~dB}$ lower transmitted power by the device (lower SAR), due to power control. These different macrocell scenarios then each represent a building located closer or further from a UMTS macrocell. Uniformly distributed transmit power values of $-50,-30,-10$, and $10 \mathrm{dBm}$ will be simulated, together with the corresponding received power (electric-field) values. This total of four different values represent four different locations of the macrocell relative to the considered buildings. The transmitted and received powers, 


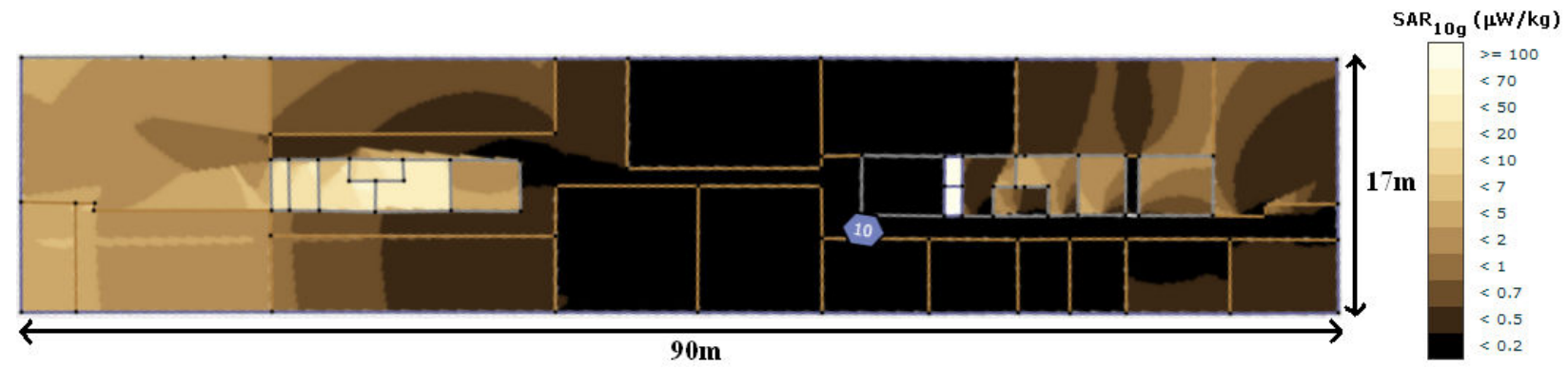

Fig. 1. Localized $S A R_{10 g}$ during a phone call at the different locations in the considered office building for UMTS femtocell scenario (femtocell base station $=$ hexagon $)$.

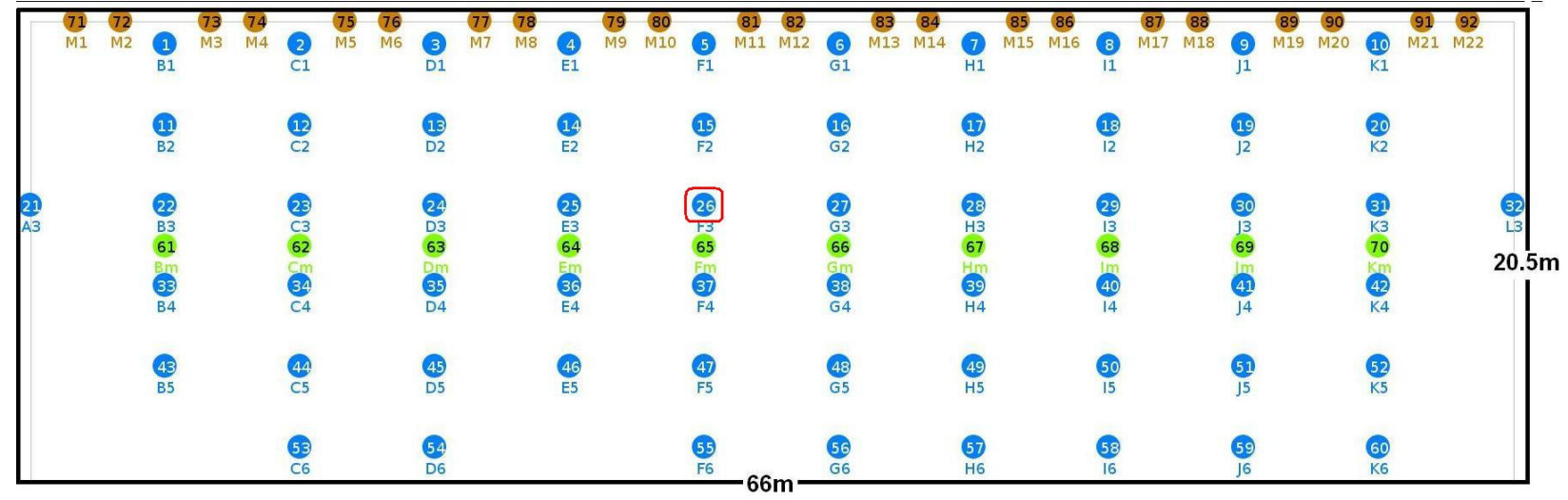

Fig. 2. The considered test lab for UMTS femtocell scenario (femtocell base station and WiFi node location = node 26, circled in red). The testbed nodes are indicated with the blue dots.

related by the UMTS power control mechanism, of these scenarios are summarized in Table II and were obtained based on measurements in [4]. The four macrocell scenarios were chosen to have a set of configurations with varying DL (and UL) exposure characteristics. Macro 1 represents a macrocell scenario where the base station is located relatively close to the considered building (good connection), while the scenarios with higher index numbers represent situations where the path loss between the macrocell and the building is progressively higher (worse connection, e.g., due to higher distances and/or more obstacles between the macrocell and the building).

\begin{tabular}{|l|r|r|r|r|}
\hline scenario & $\begin{array}{r}\mathrm{P}_{\mathrm{Tx}} \\
{[\mathrm{dBm}]}\end{array}$ & $\begin{array}{r}\mathrm{SAR}_{10 \mathrm{~g}} \\
{[\mu \mathrm{W} / \mathrm{kg}]}\end{array}$ & $\begin{array}{r}\mathrm{P}_{\mathrm{Rx}} \\
{[\mathrm{dBm}]}\end{array}$ & $\begin{array}{r}\mathrm{E} \\
{[\mathrm{mV} / \mathrm{m}]}\end{array}$ \\
\hline \hline Macro 1 & -50 & 0.021 & -35 & 270 \\
\hline Macro 2 & -30 & 2.1 & -55 & 27 \\
\hline Macro 3 & -10 & 210 & -75 & 2.7 \\
\hline Macro 4 & 10 & $2.1 \cdot 10^{4}$ & -95 & 0.27 \\
\hline
\end{tabular}

TABLE II

FOUR MACROCELL SCENARIOS WITH DEVICE TRANSMIT POWER P $\mathrm{P}_{\text {Tx }}$, $\mathrm{SAR}_{10 \mathrm{~g}}$ VALUE, RECEIVED POWER $\mathrm{P}_{\mathrm{Rx}}$, AND RECEIVED ELECTRIC-FIELD STRENGTH E.

\section{A. Dowlink: electric-field strength $E$}

Fig. 3 and Table III compare the DL electric-field distributions of the different scenarios for the two environments. The lower field strengths in the WiFi case compared to the UMTS femtocell case (approximately a factor 7, both in the office and the test lab environment) are mainly due to the use of a duty cycle in WiFi communication. Due to the more open environment, the test lab environment has higher electricfield strengths than the test lab environment (from a factor 3.5 for the $25 \%$-percentile to a factor 2.3 for the $75 \%$-percentile, see Fig. 3 and Table III). Table II shows the field strength percentile values for the macrocell scenarios. There is for each scenario only one value for all percentiles, due to the assumption of a uniform distribution of the field values in the building. Only Macro 4 (bad connection) has a lower DL exposure than the WiFi office scenario. Macro 3 causes higher DL exposure than the WiFi office scenario, but lower exposure than the WiFi test lab scenario. Due to the vicinity of the indoor base station, the femtocell scenarios (office and test lab) cause a relatively high DL exposure, but Macro 1 still causes the highest DL exposure.

\section{B. Uplink: localized $\mathrm{SAR}_{10 \mathrm{~g}}$}

Fig. 1 shows the $\mathrm{SAR}_{10 \mathrm{~g}}$ distribution in the office building for the UMTS femtocell scenario. It is observed that due to power control, the locations closer to the FBS have 


\begin{tabular}{|r|r|r|r|}
\hline downlink DL & $\begin{array}{r}\mathbf{E}^{\mathbf{2 5}} \\
{[\mathbf{V} / \mathbf{m}]}\end{array}$ & $\begin{array}{r}\mathbf{E}^{\mathbf{5 0}} \\
{[\mathbf{V} / \mathbf{m}]}\end{array}$ & $\begin{array}{r}\mathbf{E}^{\mathbf{7 5}} \\
{[\mathbf{V} / \mathbf{m}]}\end{array}$ \\
\hline WiFi office & $1.0 \cdot 10^{-3}$ & $1.6 \cdot 10^{-3}$ & $3.7 \cdot 10^{-3}$ \\
\hline Femto office & $7.1 \cdot 10^{-3}$ & $1.1 \cdot 10^{-2}$ & $2.6 \cdot 10^{-2}$ \\
\hline \hline WiFi test lab & $3.5 \cdot 10^{-3}$ & $5.1 \cdot 10^{-3}$ & $8.4 \cdot 10^{-3}$ \\
\hline Femto test lab & $2.5 \cdot 10^{-2}$ & $3.6 \cdot 10^{-2}$ & $5.9 \cdot 10^{-2}$ \\
\hline \hline uplink UL & $\begin{array}{r}\mathbf{S A R}_{\mathbf{1 0 g}}^{\mathbf{2 5}} \\
{[\mathbf{W} / \mathbf{k g}]}\end{array}$ & $\begin{array}{r}\mathbf{S A R}_{\mathbf{1 0 g}}^{\mathbf{5 0}} \\
{[\mathbf{W} / \mathbf{k g}]}\end{array}$ & $\begin{array}{r}\mathbf{S A R}_{\mathbf{1 0}}^{\mathbf{7 5}} \\
{[\mathbf{W} / \mathbf{k g}]}\end{array}$ \\
\hline WiFi office & $9.8 \cdot 10^{-4}$ \\
\hline Femto office & $6.8 \cdot 10^{-8}$ & $3.6 \cdot 10^{-7}$ & $9.2 \cdot 10^{-7}$ \\
\hline \hline WiFi test lab & \multicolumn{3}{|c|}{$9.8 \cdot 10^{-4}$} \\
\hline Femto test lab & $1.3 \cdot 10^{-8}$ & $3.6 \cdot 10^{-8}$ & $7.5 \cdot 10^{-8}$ \\
\hline
\end{tabular}

TABLE III

25\%-, 50\%-, AND 75\%-PERCENTILES OF FIELD STRENGTH E AND $\mathrm{SAR}_{10 \mathrm{~g}}$ FOR UMTS FEMTOCELL AND WIFI AP SCENARIO IN OFFICE AND TEST LAB ENVIRONMENT.

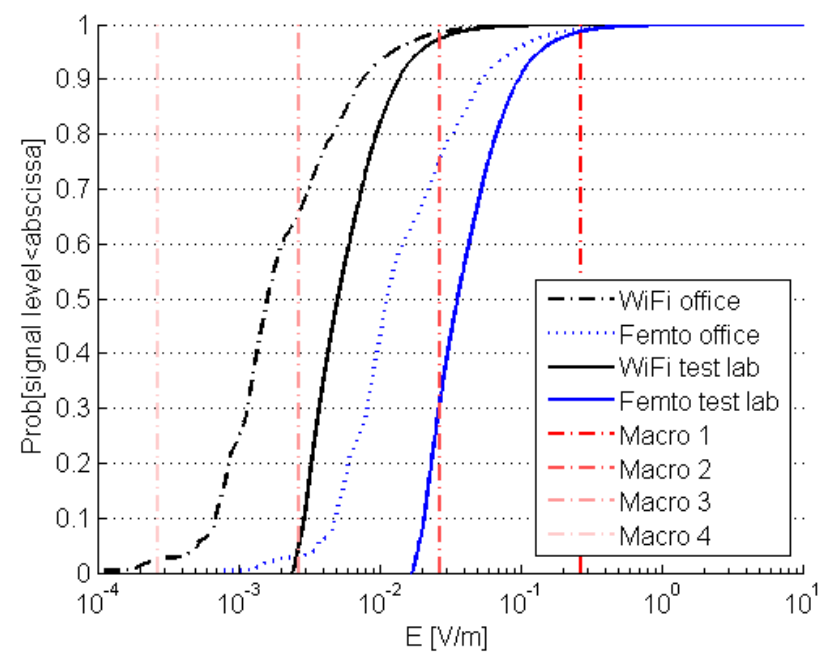

Fig. 3. E-distribution in the office and test lab buildings for WiFi AP scenario, UMTS femtocell scenario, and four UMTS macrocell scenarios.

lower transmit powers and thus lower $\mathrm{SAR}_{10 \mathrm{~g}}$ values. Fig. 4 compares the $\mathrm{SAR}_{10 \mathrm{~g}}$ distributions of the different scenarios, and percentile values are listed in Table III for the UMTS femtocell and WiFi AP scenario. Fig. 4 and Table III show that, due to the constant mobile phone UL power of $20 \mathrm{dBm}$ in the WiFi scenario, the 25\%-, 50\%-, and 75\%-percentile values are the same, irrespective of the environment (office or test lab). They also show that due to the power control mechanism, the localized SAR values in the UMTS femtocell scenario are noticeably lower (i.e., 2722 times for office and 27222 times for test lab) than in the WiFi AP scenario. Table II shows the $\mathrm{SAR}_{10 \mathrm{~g}}$ percentile values for the macrocell scenarios. The femtocell test lab scenario is comparable to the Macro 1 scenario (best macrocell connection), the femtocell office scenario is in between Macro 1 and Macro 2. With respect to the two femtocell scenarios, $\mathrm{SAR}_{10 \mathrm{~g}}$ values are higher for the office scenario than for the test lab scenario (around a factor 10, due to the worse connection, on average).
The WiFi scenario causes a relatively high UL exposure $(0.98 \mathrm{~mW} / \mathrm{kg})$, with values only exceeded by Macro 4 (the worst macrocell connection scenarios).

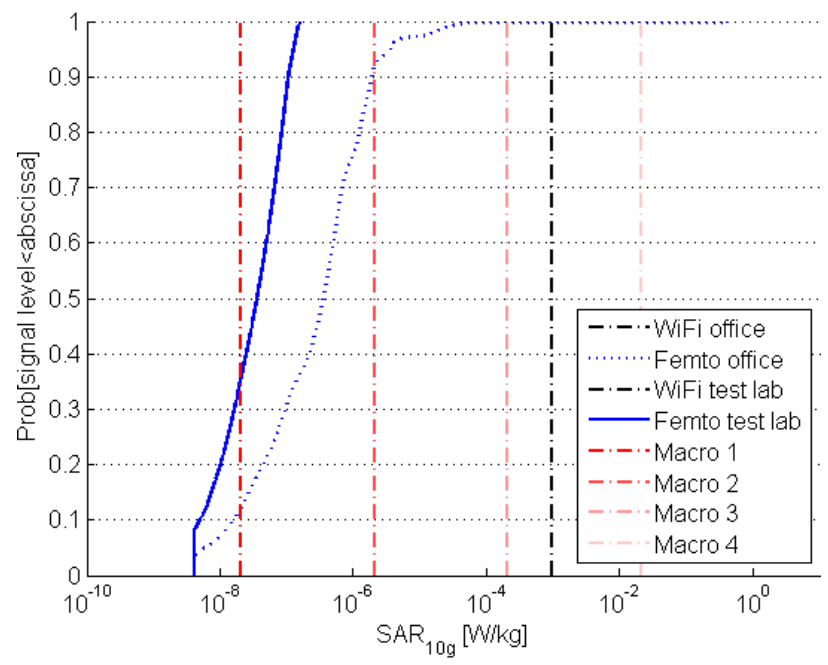

Fig. 4. Localized $\mathrm{SAR}_{10 \mathrm{~g}}$ distribution in the office and test lab buildings for WiFi AP scenario, UMTS femtocell scenario, and four UMTS macrocell scenarios.

\section{Discussion}

Comparison of two random scenarios in Figs. 3 and 4 shows that in general, that scenarios with lower DL exposure (E) result in higher UL absorption $\left(\mathrm{SAR}_{10 \mathrm{~g}}\right)$. E.g., the WiFi scenarios cause lower electric-field strengths than the UMTS femtocell scenarios, but the latter ones cause lower $\mathrm{SAR}_{10 \mathrm{~g}}$ values due to an efficient power control. Logically, in the macrocell scenarios providing a better connection to the MP (e.g., Macro 1), higher field strengths but lower $\mathrm{SAR}_{10 \mathrm{~g}}$ values are observed than in the macrocell scenarios with a worse connection (e.g., Macro 4). Some conclusions can be drawn with respect to the 'best' scenario from an exposure point of view.

In case locations in the building have a good connection with the macrocell base station (e.g., Macro 1), it is better to rely on the macrocell, as it(s downlink exposure) is present anyway and due to the good connection, low $\mathrm{SAR}_{10 \mathrm{~g}}$ values are observed when making a phone call. However, if the macrocell is not able to provide an excellent connection with the device inside the building (e.g., Macro 2), the use of a femtocell might be a better choice, especially when the user calls a lot (lower (uplink) exposure doses in the femtocell case). If the macrocell is located further from the building (worse connection with macrocell, e.g. Macro 4), femtocells are always the best choice when the users call duration is long, due to the advantageous UMTS power control mechanisms when the user is close to the femtocell base station. For short phone call durations, one could expect that the network deployer could either rely on the existing macrocell infrastructure or either add a WiFi access point, due to the lower exposure due to the base station 
(compared to a femtocell deployment). However, the wholebody and localized exposure doses due to the mobile device operating at higher power during even a very short time, already exceeds the exposure doses due to the continuously present (and nearby) femtocell base station.

As a summary, we can state that the use of a femtocell becomes advantageous when the connection with the macrocell is deteriorating. From an exposure point-of-view, the use of a WiFi deployment is never the best solution, although it has the advantage of also allowing data traffic besides voice traffic. It must be noted that all these remarks are based on current deployments, with macrocell networks planned to also provide indoor coverage. Results quantifying the statements in this discussion can be found in [4].

\section{CONCLUSIONS}

In this paper, a downlink electric-field and uplink SAR prediction algorithm in a wireless network planner is presented. It allows calculating both whole-body exposure due to base stations or access points (downlink exposure) and localized exposure due to the mobile device (uplink exposure) in indoor wireless networks. Three phone call scenarios are investigated (UMTS macrocell, UMTS femtocell, and WiFi voice-over-IP) and they are compared on the level of electricfield strength and localized $\mathrm{SAR}_{10 \mathrm{~g}}$ distributions for two building types (office and test lab). The benefit of a low localized $\mathrm{SAR}_{10 \mathrm{~g}}$ due to the UMTS power control mechanism is illustrated, but dependent on the connection quality with the existing macrocells, also the macrocell solution might be preferential. This paper paves the way for further research, in which predictions and numerical comparisons of exposure doses (accounting for the a users average daily phone call duration) will be performed.

\section{ACKNOWLEDGEMENT}

This work has been carried out with the financial support of the iMinds-RAILS and FP7-LEXNET projects. W. Joseph is a Post-Doctoral Fellow of the FWO-V (Research FoundationFlanders).

\section{REFERENCES}

[1] R.P. Torres, L. Valle, M. Domingo, M.C. Diez, "CINDOOR: an engineering tool for planning and design of wireless systems in enclosed spaces," IEEE Antennas and Propagation Magazine, vol. 41, no. 4, pp. 11-22, Sept. 1999.

[2] Aerohive Networks, "Aerohive Wi-Fi Planning Tool," Website, http://www.aerohive.com/build-your-network.

[3] D. Plets, W. Joseph, K. Vanhecke, E. Tanghe, and L. Martens, "Coverage Prediction and Optimization Algorithms for Indoor Environments," EURASIP Journal on Wireless Communications and Networking, Special Issue on Radio Propagation, Channel Modeling, and Wireless, Channel Simulation Tools for Heterogeneous Networking Evaluation, vol. 1, 2012. [Online]. Available: http://jwcn.eurasipjournals.com/content/2012/1/123

[4] D. Plets, W. Joseph, S. Aerts, K. Vanhecke, and L. Martens, "Prediction and Comparison of Downlink Electric-Field and Uplink Localized SAR Values for Realistic Indoor Wireless Network Planning," Radiation Protection Dosimetry, published.
[5] "Federal Communications Commission. Office of Engineering and Technology." Tech. Rep., last accessed on Dec 19, 2013. [Online]. Available: url = https://apps.fcc.gov/ oetcf/eas/reports/ViewExhibitReport.cfm?mode= Exhibits\&calledFromFrame=N\&application_id $=922267$

[6] "Federal Communications Commission. Office of Engineering and Technology." Tech. Rep., last accessed on Dec 19, 2013. [Online]. Available: url = https://apps.fcc.gov/ oetcf/eas/reports/ViewExhibitReport.cfm?mode= Exhibits\&calledFromFrame $=$ N\&application_id $=456890$

[7] D. Plets, W. Joseph, K. Vanhecke, and L. Martens, "Exposure Optimization in Indoor Wireless Networks by Heuristic Network Planning," Progress In Electromagnetic Research (PIER), vol. 139, pp. 445-478, 2013.

[8] W. Joseph, D. Pareit, G. Vermeeren, D. Naudts, L. Verloock, L. Martens, and I. Moerman, "Determination of the duty cycle of WLAN for realistic radio frequency electromagntic field exposure assessment," Progress in Biophysics \& Molecular Biology, October 2012.

[9] A. Gati, E. Conil, M.-F. Wong, and J. Wiart, "Duality between uplink local and downlink whole-body exposures in operating networks," Electromagnetic Compatibility, IEEE Transactions on, vol. 52, no. 4, pp. 829-836, 2010.

[10] S. Aerts, D. Plets, L. Verloock, W. Joseph, and L. Martens, "Assessment and Comparison of RF EMF Exposure in Femtocell and Macrocell Scenarios," Bioelectromagnetics, accepted. 